\section{Reporte de notas de actividad física en Chile: Contribuyendo en una iniciativa global por niños y adolescentes más activos}

\section{Chile's report card on physical activity: contributing to a global initiative for more active children and adolescents}

\section{Sr. Editor:}

Recientemente, se ha conformado una red de investigación internacional denominada "Active Healthy Kids Global Alliance" (AHKGA) ${ }^{1}$, la que tiene por finalidad sintetizar la mejor evidencia científica disponible sobre una serie de indicadores (comportamiento individual, niveles de influencia, estrategias e inversión económica) relacionados con los niveles de actividad física en niños y adolescentes. Chile participará por primera vez en la segunda versión de esta matriz global que incluye el reporte de 38 naciones, la que será interpretada y evaluada por un comité de expertos de diferentes países y será publicada el presente año.

Los dominios a evaluar dentro de esta matriz son: 1) Actividad física global; 2) Transporte activo; 3) Participación en deportes; 4) Juego activo; 5) Conducta sedentaria; 6) Sobrepeso y obesidad; 7) Condición física; 8) Entorno familiar y pares; 9) Entorno escolar; 10) Comunidad y medio ambiente y 11) Políticas públicas. Después de evaluar la evidencia disponible a nivel nacional en cada una de estas áreas, el comité procederá a otorgar una calificación basada en el grado de cumplimiento en cada dominio, apoyándose en las metas establecidas por AHKGA, tal como se hizo en su primera versión ${ }^{1}$.

De esta forma, el reporte de notas de actividad física infantil, tanto a nivel nacional como internacional, tendrá el potencial de transformarse en una herramienta de comunicación y defensa ("advocacy") basada en la evidencia para liderar la acción social e inspirar futuros cambios sociales en la promoción de la actividad física ${ }^{2}$, siendo un ejemplo de traslación del conocimiento en el área de la salud pública ${ }^{3}$. El reporte de notas pretenderá contribuir a: 1) Desarrollar mecanismos de concientización pública para llamar a la acción a través de una estrategia de abogacía nacional; 2) Proveer indicadores de la situación actual del país; 3) Implementar un sistema de vigilancia epidemiológica; 4) Servir como referencia para que líderes y organizaciones promuevan actividad física; 5) Ser una guía para el desarrollo de políticas; 6) Facilitar la identificación de necesidades de investigación; 7) Estimular a otros profesionales, regiones o países, con el fin de implementar procesos similares para permitir comparaciones y facilitar mejoras en otras temáticas relevantes para la salud ${ }^{1}$.

De la primera versión realizada con 15 países en el año 2014, el hallazgo más notable de la matriz global fue la variación sustancial en las notas asignadas a los indicadores de actividad física entre las distintas naciones. Este aspecto fue un gran motivador para los investigadores dado que, por una parte, se demostró que algunos países están siendo exitosos en cada uno de los indicadores evaluados en la matriz global. Por otra parte, dicha variación internacional presenta una oportunidad para la proliferación cruzada de ideas con el fin de mejorar los indicadores en cada uno de los países participantes. Y, finalmente, la matriz global aportó un marco conceptual sólido para dirigir la investigación en base a las necesidades de desarrollo detectadas en cada uno de los países participantes ${ }^{1}$.

Es importante destacar que la participación de estos 38 países en la matriz global de actividad física infantil, representará aproximadamente $20 \%$ de los países del mundo, abarcando 40\% del área total del planeta y $60 \%$ de la población mundial. Por lo tanto, la información contenida en este proyecto colaborativo internacional será de gran impacto en nuestro país. Paralelamente, se están generando redes de colaboración internacional en el área de la actividad física y salud pública de forma creciente en el mundo, con el fin de coordinar acciones locales y globales de cambio, como lo son la presente iniciativa y The Lancet Physical Activity Observatory, que está enfocada en el reporte de la población adulta ${ }^{4}$.

Por consiguiente, es importante situar a nuestro país en un contexto global que permita contribuir, a través de evidencia científica de calidad, a identificar fortalezas y debilidades relacionadas con la práctica de actividad física en niños y adolescentes, con el objetivo de ayudar a direccionar las estrategias de investigación e implementación de programas y políticas públicas en nuestro país. Finalmente, la existencia de un reporte y actualización sistemática de la información en nuestro país, 
será un instrumento extremadamente útil para la elaboración y adaptación de recomendaciones de actividad física para la población chilena, tal como lo está realizando actualmente la convocatoria de los Ministerios de Salud, Deporte y Educación.

Nicolás Aguilar-Farías ${ }^{1}$, Andrea Cortínez ${ }^{2,3}$, Jaime Leppe-Zamora ${ }^{4}$, Teresa Balboa, Carolina Cobos ${ }^{6}$, Nicolás Lemus ${ }^{7,8}$, Macarena Valladares ${ }^{9}$, Kabir Sadarangani ${ }^{10}$, Astrid Von Oetinger ${ }^{11}$, Magdalena Walbaum ${ }^{12}$, Carlos Cristi-Montero ${ }^{13}$

${ }^{1}$ Departamento de Educación Física, Deportes y Recreación, Universidad de La Frontera, Temuco, Chile

${ }^{2}$ Laboratorio de Cambio Social, Departamento de Ingeniería de Transportes y Logística, Pontificia

Universidad Católica de Chile. Santiago, Chile.

${ }^{3}$ Centro de Desarrollo Urbano Sustentable

(CEDEUS). Pontificia Universidad Católica de Chile.

${ }^{4}$ Carrera de Kinesiología, Facultad de Medicina Clínica Alemana-Universidad del Desarrollo. Santiago, Chile.

${ }^{5}$ Departamento de Salud Pública, Universidad de La Frontera, Temuco, Chile.

${ }^{6}$ Departamento de Promoción de la Salud y Participación Ciudadana, División de Políticas Públicas Saludables y Promoción, Ministerio de Salud, Chile.

${ }^{7}$ Unidad de Currículum y Evaluación, Ministerio de Educación, Chile. ${ }^{8}$ Universidad Mayor, Chile. ${ }^{9}$ Unidad de Salud del Observatorio Regional de Paz y Seguridad (ORPAS), Universidad Bernardo O’Higgins. Santiago, Chile.
${ }^{10}$ Escuela de Kinesiología, Facultad de Ciencias de la Salud, Universidad San Sebastián, Chile.

${ }^{11}$ Escuela de Kinesiología, Facultad de Ciencias de la Rehabilitación, Universidad Andrés Bello, Chile.

${ }^{12}$ Departamento de Enfermedades no Transmisibles, División de Prevención y Control de Enfermedades, Subsecretaría de Salud Pública, Ministerio de Salud, Chile. ${ }^{13}$ Grupo IRyS. Escuela de Educación Física. Pontificia Universidad Católica de Valparaíso, Chile.

\section{Referencias}

1. Tremblay MS, Gray CE, Akinroye K, Harrington DM, Katzmarzyk PT, Lambert EV, et al. Physical activity of children: a global matrix of grades comparing 15 countries. J Phys Act Health 2014; 11 (Suppl 1): S113-25.

2. Tremblay MS, Barnes JD, Cowie Bonne J. Impact of the Active Healthy Kids Canada report card: a 10-year analysis. J Phys Act Health 2014; 11 (Suppl 1): S3-S20.

3. Colley RC, Brownrigg M, Tremblay MS. A model of knowledge translation in health: the Active Healthy Kids Canada Report Card on physical activity for children and youth. Health Promot Pract 2012; 13 (3): 320-30.

4. Hallal PC, Martins RC, Ramírez A. The Lancet Physical Activity Observatory: promoting physical activity worldwide. Lancet 2014; 384 (9942): 471-2. 